\title{
Persistência na lactação para vacas da raça Holandesa criadas no Estado do Rio Grande do Sul via modelos de regressão aleatória
}

\author{
Lactation persistency for Holstein cows raised in the State of Rio Grande do Sul \\ using a random regression model
}

\section{Cristian Kelen Pinto Dorneles ${ }^{\mathrm{I}}$ Paulo Roberto Nogara Rorato ${ }^{\mathrm{II}}$ Jaime Araújo Cobuci ${ }^{\mathrm{II}}$ Jader Silva Lopes ${ }^{\mathrm{I}}$ Tomás Weber $^{\mathrm{I}}$ Henrique Nunes de Oliveira ${ }^{\mathrm{IV}}$}

\section{RESUMO}

Foram utilizados 21.702 registros de produção de leite no dia do controle de 2.429 vacas primíparas da raça Holandesa, filhas de 233 touros, coletados em 33 rebanhos do Estado do Rio Grande do Sul, entre 1992 e 2003, para estimar parâmetros genéticos, para três medidas de persistência (PS1, PS2 e PS3) e para a produção de leite até 305 dias (P305) de lactação. Os modelos de regressão aleatória ajustados aos controles leiteiros entre o sexto e o 300 dia de lactação incluíram o efeito de rebanho-ano-mês do controle, a idade da vaca ao parto e os parâmetros do polinômio de Legendre de ordem quatro, para modelar a curva média da produção de leite da população e os parâmetros do mesmo polinômio, para modelar os efeitos aleatórios genético-aditivo direto e de ambiente permanente. As estimativas de herdabilidade obtidas foram 0,05, 0,08 e 0,19, respectivamente, para PS1, PS2 e PS3 e 0,25, para P305 sugerindo a possibilidade de ganho genético por meio da seleção para PS3 e para P305. As correlações genéticas entre as três medidas de persistência e P305, variaram de -0,05 a 0,07, indicando serem persistência e produção, características determinadas por grupos de genes diferentes. Assim, consequentemente, a seleção para P305, geralmente praticada, não promove progresso genético para a persistência.

Palavras-chave: correlação genética, herdabilidade, método REML, polinômio de Legendre, produção de leite no dia do controle, seleção.

\section{ABSTRACT}

There were used 21,702 test day milk yields from 2,429 first parity Holstein breed cows, daughters of 2,031 dams and 233 sires, distributed over 33 herds in the state of Rio
Grande do Sul, from 1992 to 2003. Genetic parameters for three measures of lactation persistency (PS1, PS2 e PS3) and for milk production to 305 days (P305) were evaluated. A random regression model adjusted by fourth order Legendre polynomial was used. The random regression model adjusted to test day between the sixth and the $305^{\text {th }}$ lactation day included the herd-year-season of the test day, the age of the cow at the parturition effects and the order fourth Legendre polinomial parameters, for modeling the milk production average curve of the population, and parameters of the same polinomial for modeling the random additive genetic and permanent environmental effects. The estimated heritabilities were 0.05, 0.08 and 0.19 , respectively to PS1, PS2 and PS3, and 0.25 to P305, suggesting the possibility of a genetic gain by selection for PS3 and P305. The genetic correlations between persistency measurements and P305 ranged from -0.05 to 0.07, suggesting being, persistency and milk yield, characteristics determined by different gene groups, and that the selection for P305, usually done, do not promote genetic progress for persistency.

Key words: genetic correlations, heritability, Legendre polynomial, REML method, selection test day.

\section{INTRODUÇÃO}

A atividade leiteira no Brasil, historicamente, vem sendo caracterizada por períodos em que o produtor recebe remuneração adequada, que lhe proporciona retorno financeiro, seguidos por outros em que é forçado a adequar o custo de produção ao valor pago pela indústria, via restrição alimentar, fazendo com que, muitas

IPrograma de Pós-graduação em Zootecnia, Departamento de Zootecnia, Universidade Federal de Santa Maria (UFSM), Santa Maria, RS, Brasil.

IIDepartamento de Zootecnia, UFSM, Campus Universitário, Bairro Camobi, 97105-900, Santa Maria, RS, Brasil. E-mail: rorato@smail.ufsm.br. Autor para correspondência.

IIIDepartamento de Zootecnia, Universidade Federal do Rio Grande do Sul (UFRGS), Porto Alegre, RS, Brasil.

${ }^{\text {IV }}$ Departamento de Zootecnia, Universidade Estadual Paulista (UNESP), Campus de Botucatu, Botucatu, SP, Brasil. 
vezes, esse produtor abandone a atividade. Apesar dessa realidade, a produção de leite vem crescendo a cada ano, talvez por ser uma atividade que assegure ao produtor uma remuneração mensal no decorrer do ano. Segundo a FAO (2007), a produção de leite no Brasil apresentou crescimento próximo a 37\% nos últimos 10 anos. Todavia, a produtividade média de nossos rebanhos leiteiros deixa muito a desejar, variando de 3,22 litros/vaca/dia na região Norte a 7,05 litros vaca-1 $\mathrm{dia}^{-1}$ na região Sul, com média Nacional de 5,61 litros vaca-1 $\mathrm{dia}^{-1}$ (ANUALPEC, 2008). Portanto, é necessário que providências sejam tomadas no sentido de aumentar a produção, bem como a produtividade, lançando mão de programas de melhoramento genético, de melhorias no manejo, além da formulação de políticas de comercialização que estimulem os produtores a permanecerem na atividade.

Uma forma eficiente de avaliar o desempenho produtivo de uma vaca leiteira é por meio do estudo da curva da lactação, que é a representação gráfica da produção de leite no decorrer da lactação. O estudo das curvas de lactação permite ajustar o manejo geral dos rebanhos leiteiros, principalmente o nutricional, e identificar as causas de baixa produção, além de ser um instrumento importante para viabilizar a gestão de fazendas leiteiras, pois permite estabelecer a variação esperada na produção de leite.

A persistência na lactação o pico e a duração da lactação foram consideradas as principais características para descrever a curva de lactação por SANDERS (1930) epor LUDWICK \& PETERSEN(1943) e, em seguida, como o principal componente da curva de lactação por WOOD (1967). Para MUIR et al. (2004), persistência é a maneira como a produção de leite se mantém por toda a lactação, calculada como a quantidade atual de leite em relação à quantidade do mês anterior, em porcentagem FREITAS (2008) definiu persistência como a habilidade de uma fêmea manter níveis mais constantes de produção até o final da lactação. Apesar de existirem na literatura inúmeras definições para persistência na lactação, ainda não há um consenso da melhor maneira de expressar essa característica em termos matemáticos (COLE \& VAN RADEN, 2006); via de regra, a persistência na lactação é definida como a capacidade da vaca em manter a produção de leite após atingir sua produção máxima na lactação. GENGLER (1996) definiu como mais persistente a vaca que, comparada com outra de produção equivalente e em período similar, apresenta o pico da curva de produção mais baixo e, por conseguinte, uma curva de lactação mais achatada, resultado da distribuição mais equilibrada da produção de leite no decorrer da lactação.
A persistência na lactação está diretamente relacionada a aspectos econômicos da atividade leiteira e à melhoria desta pode contribuir para a redução de custos no sistema de produção (TEKERLI et al., 2000; JAKOBSEN et al., 2002). Ao avaliarem os aspectos econômicos relacionados com a persistência, DEKKERS et al. $(1996,1998)$ relataram que o valor econômico dessa característica é influenciado pelo custo com alimentação, com saúde e com reprodução animal, bem como pelo retorno econômico obtido pela produção adicional de leite, devido à melhoria do nível de persistência na lactação dos animais.

Dentre os métodos usados no cálculo das diferentes medidas de persistência, os modelos de regressão aleatória (MRA) têm sido indicados como uma das melhores alternativas, pois permitem a predição do valor genético dos animais em qualquer período de lactação, fornecendo, assim, um ótimo mecanismo para estudos da persistência (LIN \& TOGASHI, 2002). Além disso, permitem uma avaliação mais precisa dos efeitos ambientais que atuam sobre essa característica (STRABEL \& JAMROZIK, 2002), o que pode melhorar a acurácia das avaliações genéticas (LIN \& TOGASHI, 2002).

O objetivo do presente estudo foi estimar, por meio do modelo de regressão aleatória, parâmetros genéticos para três medidas de persistência na lactação e para a produção de leite em 305 dias, bem como correlações genéticas entre as medidas de persistência e a produção, para vacas da raça Holandesa no Estado do Rio Grande do Sul.

\section{MATERIAL E MÉTODOS}

Os dados utilizados no presente estudo são provenientes dos arquivos do Serviço de Controle Leiteiro da Associação de Criadores de Gado Holandês do Rio Grande do Sul (ACGHRS), com sede em Porto Alegre, Rio Grande do Sul (RS).

O arquivo inicial continha 168.103 registros de produção de leite no dia do controle. As vacas apresentaram várias ordens de lactação, os dados foram coletados mensalmente pela ACGHRS entre os anos de 1991 e 2003, em 33 rebanhos distribuídos em diferentes regiões do RS.

Para aumentar a consistência do conjunto de dados, foram eliminadas as informações oriundas de vacas com idade ao primeiro parto inferior a 20 e superior a 48 meses; vacas filhas de touros que não tivessem no mínimo uma filha em dois rebanhos; vacas que não tivessem no mínimo seis controles de produção de leite na lactação e que esses controles não tivessem sido realizados entre o sexto e o $305^{\circ}$ dia de lactação; 
registros de produções diárias de leite inferiores a seis e superiores a $37 \mathrm{~kg}$; bem como animais que não fossem puros de origem (PO). Restaram 21.702 registros de produção de leite no dia do controle de 2.429 vacas primíparas da raça Holandesa, filhas de 233 touros, coletados em 33 rebanhos, entre os anos de 1992 e 2003.

Na predição dos valores genéticos dos animais, para persistência na lactação e produção de leite até 305 dias, utilizou-se o seguinte modelo de regressão aleatória:

$$
\begin{aligned}
& y_{i j k}=R A M C_{i}+\sum_{l=1}^{2} b_{l} x_{i j k}+\sum_{m=1}^{4} q_{m} Z_{j k m}+\text {,em que } \mathrm{y}_{\mathrm{ijk}} \\
& \sum_{m=1}^{4} a_{j m} Z_{j k m}+\sum_{m=1}^{4} p_{j m} Z_{j k m}+e_{i j k}
\end{aligned}
$$

é o controle $k$ da vaca $j$, num período qualquer de dias em lactação (t), dentro da classe i de rebanho-ano-mês do controle leiteiro; $\mathrm{RAMC}_{\mathrm{i}}$ é o efeito fixo de rebanhoano-mês do controle leiteiro; $b_{1}$ e $b_{2}$ são os coeficientes de regressão linear e quadrático da produção de leite sobre idade da vaca no parto; $x_{i j k}$ é a idade da vaca no parto, em meses; $q_{m}$ é o vetor dos coeficientes fixos da regressão específicos para a modelagem da curva de lactação média da população; $a_{j m}$ e $p_{j m}$, são vetores dos coeficientes aleatórios da regressão específicos para a vaca j; $Z_{j k m}$ é o vetor de covariáveis, representado pelo polinômio de Legendre, em que $m$ representa o $m$ ésimo parâmetro do polinômio de Legendre; $e_{i j k}$ é o efeito aleatório residual associado a $y_{i j k}$. O polinômio de Legendre de ordem quatro foi usado, tanto para as regressões fixas, como para as regressões aleatórias, uma vez que, segundo ARAÚJO et al. (2006), são necessárias funções de covariância com polinômio de quarta ordem, no mínimo, para descrever a variação genética da produção de leite. A variância residual foi considerada constante ao longo do período de lactação, assim como consideraram COBUCI et al. (2004), ARAÚJO et al. (2006) e DIONELLO et al. (2006).

As matrizes de (co)variâncias dos coeficientes de regressão (genético-aditivo e de ambiente permanente), necessárias para o cálculo dos parâmetros genéticos e dos valores genéticos, foram obtidas por meio do programa DxMRR (MEYER, 1998). Definiu-se como critério de convergência a diferença entre o valor de -2log da função de verossimilhança, obtido em iterações consecutivas, menor que $10^{-9}$.

As estimativas de covariância genética, $\hat{\sigma}_{a}^{2} \mathrm{e}$ de ambiente permanente, $\hat{\sigma}_{a p}^{2}$, entre dois controles, em um período $t$ qualquer da lactação, foram obtidas por:

$$
\begin{aligned}
& \hat{\sigma}_{a\left(t^{\prime} t\right)}^{2}=Z_{t^{\prime}}^{\prime} \hat{G} Z_{t} \\
& \hat{\sigma}_{a p\left(t^{\prime} t\right)}^{2}=Z_{t^{\prime}}^{\prime} \hat{P} Z_{t}
\end{aligned}
$$

em que $\mathrm{G}$ e $\hat{P}$ representam, respectivamente, as matrizes de (co)variâncias genética e de ambiente permanente entre os coeficientes de regressão aleatória, Zt é a matriz de covariáveis e Z't' é transposto de Zt, para t' $\neq$ t. Assim, os componentes de variância foram dados quando $\mathrm{t}^{\prime}=\mathrm{t}$.

As estimativas de herdabilidade para as medidas de persistência na lactação (PS) e produção de leite até 305 dias (P305) foram obtidas, conforme JAKOBSEN et. al. (2002) por

$$
\begin{aligned}
& \hat{h}^{2}{ }_{(P S i)}=\frac{\hat{\sigma}_{a}^{2}(i)}{\hat{\sigma}_{a}^{2}(i)+\hat{\sigma}_{e p}^{2}(i)+\left(C_{i} * \hat{\sigma}_{e}^{2}\right)} \\
& \hat{h}_{(P 305)}^{2}=\frac{\hat{\sigma}_{a}^{2}(P 305)}{\hat{\sigma}_{a}^{2}(P 305)+\hat{\sigma}_{e p}^{2}(P 305)+\left(305 * \hat{\sigma}_{e}^{2}\right)}
\end{aligned}
$$

em que $\mathrm{i}=$ PS1, PS2 e PS3 e $\mathrm{C}_{\mathrm{i}}$ é uma constante dada pelos valores 220 (produção de leite no $280^{\circ}$ dia em lactação - produção de leite no 60ำ dia em lactação), 200 e 200, respectivamente, para a i-ésima medida de persistência na lactação, PS1, PS2 e PS3.

As equações utilizadas neste estudo, para a predição dos valores genéticos dos animais, para as três medidas de persistência na lactação foram as seguintes:

$$
P S 1=\sum_{t=60}^{279}\left(V g_{t}-V g_{280}\right) \text {, a primeira medida de }
$$
persistência na lactação (PS1), foi obtida pelo somatório das contribuições de cada dia de produção de leite no período de $60^{\circ}$ a $279^{\circ}$ dia de lactação, como desvios da produção adicional aos 280 dias de lactação (JAKOBSEN et al., 2002), em que Vg é o valor genético do animal em cada dia de lactação (t);

PS $2=\left(V g_{290}-V g_{90}\right)$, a segunda medida de persistência (PS2) foi calculada pela diferença entre os valores genéticos preditos para produção de leite aos $290^{\circ}$ e $90^{\circ}$ dia de lactação (COBUCI et al., 2004)

$$
P S 3=\left(\sum_{t=101}^{300} V g_{t}-V g_{100} * 200\right) \text {, a terceira medida }
$$

de persistência (PS3), foi obtida pelo somatório dos valores genéticos do $100^{\circ}$ ao $300^{\circ}$ dia, como desvios da produção adicional aos 100 dias de lactação (Pösö, 2003 apud KISTEMAKER, 2003).

O valor genético para produção de leite em 305dias, $\mathrm{P}_{305}$, a partir da produção de leite no dia do controle, é obtido por $P_{305}=\left(\sum_{T=6}^{305} V g_{t}\right)$.

No cálculo da Eficiência Relativa da Seleção (ERS) para as medidas de persistência, utilizou-se a adaptação da fórmula proposta por FALCONER \& MACKAY (1996).

$$
E R S=\frac{\hat{r}_{a} \sqrt{\hat{h}_{P S}^{2}}}{\sqrt{\hat{h}_{P 305}^{2}}}
$$


em que $\hat{r}_{a}$ é a correlação genética aditiva entre a i-ésima medida de persistência e a produção de leite até 305 dias; $\hat{h}_{P S}^{2} \quad \hat{h}_{P 305}^{2}$ são respectivamente as estimativas de herdabilidade para a i-ésima medida de persistência e a produção de leite em até 305 dias.

\section{RESULTADOS E DISCUSSÃO}

As matrizes de (co)variâncias dos coeficientes de regressão aleatória para os efeitos genético aditivo direto e de ambiente permanente, estimados pelo modelo de regressão aleatória, estão apresentadas na tabela 1 . A estimativa de variância residual ao longo do período de lactação foi de $3,88 \mathrm{~kg}^{2}$.

As estimativas de herdabilidade para as três medidas de persistência na lactação, assim como as correlações genéticas e de ambiente permanente entre as medidas de persistência e a produção de leite até 305 dias, encontram-se na tabela 2. Observa-se que os valores de herdabilidade e das correlações foram dependentes da equação utilizada no cálculo da medida da persistência na lactação, sugerindo que alguns tipos de equação (medidas de persistência) podem ser melhores do que outros, por expressarem biologicamente determinadas alterações na produção de leite, durante o período de lactação das vacas. Estudos realizados por COBUCI et al. (2004), para avaliar a persistência na lactação de vacas da raça Holandesa no Estado de Minas Gerais, também revelaram diferenças nas estimativas de herdabilidade com o tipo de equação utilizado para cálculo da persistência na lactação. Segundo MADSEN (1975), essas diferenças podem estar relacionadas com a eficiência biológica, a eficiência estatística do tipo de mensuração da persistência e a parte da lactação utilizada no cálculo da persistência.
Os valores das estimativas de herdabilidade para as medidas de persistência foram 0,05, 0,08 e 0,19, respectivamente, para PS1, PS2 e PS3, sugerindo a possibilidade de ser obtido algum ganho genético por meio da seleção apenas para PS3. Os valores estimados para PS1 e PS2 são menores do que aqueles relatados por GENGLER et al. (1999), JAMROZIK et al. (2000), VAN DER LINDE et al. (2000), REKAYA et al. (2001), JAKOBSEN et al. (2002) e COBUCI et al. (2004), os quais variam de 0,09 a 0,30 e foram obtidos por meio de diferentes modelos de regressão aleatória e metodologias de estimação dos componentes de (co)variância.

A herdabilidade para produção de leite até 305 dias foi de 0,25 Esse valor coincide com o encontrado por FERREIRA et al. (2003), para animais da raça Holandesa no estado de Minas Gerais. Estimativas de herdabilidade obtidas para essa raça no Brasil, variando de 0,18 a 0,30, foram relatadas por FERREIRA \& FERNANDES (2000), MARION et al. (2001), FREITAS et al. (2001), BOLIGON et al. (2005), MELO et al., (2005) e WEBER et al. (2005). Contudo, esse valor é menor do que aqueles relatados por JAMROZIK \& SCHAEFFER (1997), JAKOBSEN et al. (2002) e COBUCI et al. (2004), os quais foram de 0,32, 0,42 e 0,35, respectivamente, para a raça Holandesa, no Canadá, na Dinamarca e no Brasil, utilizando modelos de regressão aleatória.

Pode-se observar (Tabela 2) que as correlações entre as medidas de persistência apresentaram valores altos. O valor de -0,98, encontrado para correlação genética entre PS1 e PS2, é semelhante ao relatado por COBUCI et al. (2004), igual a -0,99, para a raça Holandesa, no Estado de Minas Gerais.

Tabela 1 - Estimativas de (co)variância genética $(G)$ e de ambiente permanente ( $\widehat{P}$ ), para os coeficientes, obtidas pelo modelo de regressão aleatória

$\hat{G}=\left[\begin{array}{llll}\hat{g}_{11} & \hat{g}_{12} & \hat{g}_{13} & \hat{g}_{14} \\ \hat{g}_{21} & \hat{g}_{22} & \hat{g}_{23} & \hat{g}_{24} \\ \hat{g}_{31} & \hat{g}_{32} & \hat{g}_{33} & \hat{g}_{34} \\ \hat{g}_{41} & \hat{g}_{42} & \hat{g}_{43} & \hat{g}_{44}\end{array}\right]=\left[\begin{array}{rccc}4,23 & 0,31 & -0,22 & 0,15 \\ 0,31 & 0,81 & -0,13 & -0,63 \times 10^{-1} \\ -0,22 & -0,13 & 0,29 \times 10^{-1} & 0,89 \times 10^{-3} \\ 0,15 & -0,63 \times 10^{-3} & 0,89 \times 10^{-3} & 0,10\end{array}\right]$
$\hat{P}=\left[\begin{array}{llll}\hat{p}_{11} & \hat{p}_{12} & \hat{p}_{13} & \hat{p}_{14} \\ \hat{p}_{21} & \hat{p}_{22} & \hat{p}_{23} & \hat{p}_{24} \\ \hat{p}_{31} & \hat{p}_{32} & \hat{p}_{33} & \hat{p}_{34} \\ \hat{p}_{41} & \hat{p}_{42} & \hat{p}_{43} & \hat{p}_{44}\end{array}\right]=\left[\begin{array}{cccc}12,64 & -0,55 & -0,77 & 0,13 \\ -0,55 & 2,62 & -0,11 & -0,26 \\ -0,77 & -0,11 & 1,48 & -0,14 \\ 0,13 & -0,26 & -0,14 & 0,63\end{array}\right]$

Os sub índices 1, 2, 3 e 4 referem-se aos coeficientes de regressão aleatória.

Ciência Rural, v.39, n.5, ago, 2009. 
Tabela 2 - Estimativas dos coeficientes de herdabilidade (diagonal principal) e de correlações genéticas (acima da diagonal) e de ambiente permanente (abaixo da diagonal) entre as medidas de persistência (PS1, PS2 e PS3) e a produção de leite até 305 dias (P305)

\begin{tabular}{lllll}
\hline & PS1 & PS2 & PS3 & P305 \\
\hline PS1 & $\mathbf{0 , 0 5}$ & $-0,98$ & $-0,87$ & $-0,05$ \\
PS2 & $-0,97$ & $\mathbf{0 , 0 8}$ & 0,94 & 0,07 \\
PS3 & $-0,71$ & $-0,97$ & $\mathbf{0 , 1 9}$ & 0,05 \\
P305 & 0,20 & $-0,18$ & $-0,13$ & $\mathbf{0 , 2 5}$ \\
\hline
\end{tabular}

As correlações genéticas entre as medidas de persistência e a produção de leite até 305 dias apresentaram valores de baixa magnitude (variando de $-0,05$ a 0,07), o que, de acordo com JAKOBSEN et al. (2002), é desejável; caso contrário, segundo COBUCI et al. (2003), não se justificaria a realização de estudos visando à seleção para a persistência, pois bastaria que a seleção fosse feita para produção total de leite e, como consequência, o nível de persistência na lactação dos animais seria melhorado.

As correlações genéticas entre persistência na lactação e produção de leite até 305 dias, encontradas neste estudo, são próximas à estimada por JAKOBSEN et al. (2002), igual a 0,06; são inferiores à relatada por VAN DER LINDE et al. (2000), igual a 0,25; todavia estão dentro dos intervalos reportados por KISTEMAKER (2003), de -0,10 a 0,189, e por COBUCI et al. (2004), de .-0,31 a 0,55, para animais da raça Holandesa.

Segundo VAN DER LINDE et al. (2000), não há correlação entre o nível de produção e a persistência na lactação, concordando com GENGLER (1996), o qual reportou que vacas com o mesmo nível de produção de leite apresentaram diferentes níveis de persistência na lactação.

A persistência na lactação, segundo SHAHRBABAK (1997), é uma característica de importância econômica, uma vez que vacas com maior persistência consomem menor quantidade de alimento para produzir a mesma quantidade de leite produzido por uma vaca com menor persistência, tendo, por conseguinte, suas necessidades nutricionais mais facilmente supridas. Nesse sentido, COBUCI et al. (2003) afirmaram que uma maneira de produzir leite a custos menores é por meio da melhoria do nível de persistência na lactação das vacas, pois podem ser obtidos ganhos econômicos adicionais por meio do diferencial na produção de leite e da vida útil desses animais, da diminuição dos gastos com alimentação e do tratamento de doenças e da melhoria da eficiência reprodutiva dos animais.
Considerando a importância da persistência na lactação para a produtividade do sistema produtivo, foram obtidos valores para a eficiência relativa de seleção (ERS), para as três medidas de persistência. Se o critério de seleção fosse a produção de leite até 305 dias, a ERS seria de -0,022 para PS1; de 0,042 para PS2 e de 0,042 para PS3. Esses valores de eficiência, próximos de zero, são indícios de que a seleção para produção de leite não leva à melhoria da persistência na lactação e que, para obter animais com lactações mais persistentes, é necessário que a persistência seja considerada como critério de seleção.

\section{CONCLUSÕES}

Os valores estimados para a herdabilidade sugerem a possibilidade de serem obtidos ganhos genéticos por meio da seleção praticada para a terceira medida de persistência na lactação e para a produção de leite até 305 dias de lactação. Entretanto, devido à correlação genética próxima de zero entre produção e persistência, para melhorar a persistência, é necessário que esta seja incluída nos programas de melhoramento. Já para essa população, é recomendada a terceira medida de persistência, uma vez que esta apresentou o maior valor de herdabilidade e a menor associação genética com a produção de leite até 305 dias.

\section{AGRADECIMENTOS}

Os autores agradecem à Coordenação de Apoio ao Pessoal do Ensino Superior (CAPES) pelo suporte financeiro e à Associação de Criadores de Gado Holandês do Rio Grande do Sul (ACGHRS) pela concessão do banco de dados.

\section{REFERÊNCIAS}

ANUALPEC. Anuário da Pecuária Brasileira. São Paulo: FNP, 2008. 385p.

ARAÚJO, C.V. de, et al. Uso de funções ortogonais para descrever a produção de leite no dia de controle por meio de modelos de regressão aleatória. Revista Brasileira de Zootecnia, v.3, n.3, p.976-974, 2006 (supl.).

BOLIGON, A.A. et al. Herdabilidade e tendência genética para as produções de leite e de gordura em rebanhos da raça Holandesa no estado do Rio Grande do Sul. Revista Brasileira de Zootecnia, v.34, n.5, p.1512-1518, 2005. Disponível em: <http:// www.scielo.br/scielo.php?script=sci_arttext \&pid=S151635982005000500011\&lng $=$ en \&nrm=iso\&tlng $=p t>$. doi: 10.1590/S1516-35982005000500011.

COBUCI, J.A. et al. Persistência na lactação - uma revisão. Archivos Latinoamericanos Produccion Animal v.11, n.3, p.163-173, 2003.

Ciência Rural, v.39, n.5, ago, 2009. 
COBUCI, J.A. et al. Análises da persistência na lactação de vacas da raça holandesa, usando produção no dia do controle e modelo de regressão aleatória. Revista Brasileira de Zootecnia, v.33, n.3, p.546-554, 2004. Disponível em: <http:/ /www.scielo.br/scielo.php?script=sci_arttext\&pid=S1516$35982004000300004 \& \operatorname{lng}=$ en $\& n r m=i s o \& \operatorname{lng}=p t>$.

COLE, J.B.; VAN RADEN, P.M. Genetic evaluation and best prediction of lactation persistency. Journal of Dairy Science, v.89, n.7, p.2722-2728, 2006.

DEKKERS, J.C.M. et al. Genetic and economic evaluation of persistency in dairy cattle. Proc. Int. Workshop on Genetic Improvement of functional traits in cattle. Interbull Bull, v.12, p.97-102, 1996.

DEKKERS, J.C.M. et al. Economic aspects of persistency of lactation in dairy cattle. Livestock Production Science, v.53, p.237-252, 1998. Disponível em: <http://www.sciencedirect.com/ science?_ob=ArticleURL\&_udi=B6T9B-3T0SG4X$5 \&$ \&user $=687358 \&$ \&doc $=1 \&$ fmt $=\&$ \&_orig $=$ search $\&$ _s ort $=$ d \& vi e w $=$ c \&_a c ct $=$ C $000037899 \&$ \& versio $\mathrm{n}=1 \&$ _ u r l Versio n $=0 \&$ _ u s e r i d $=68735$ 8\&md5=f65293b025a6cbf3d3c70932b095898e >. Doi: 10.1016/ S0301-6226(97)00124-3.

DIONELLO, N.J.L. et al. Estimação de parâmetros genéticos utilizando-se a produção de leite no dia do controle em primeiras lactações de vacas da raça Jersey. Revista Brasileira de Zootecnia, v.35, n.4, p.1646-1652, 2006 (supl.). Disponível em: <http:// www.scielo.br/scielo.php?script=sci_arttext\&pid=S151635982006000600010\&lng=en\&nrm=iso\&tlng=pt $>$. Doi: 10.1590/ S1516-35982006000600010.

FALCONER, D.S.; MACKAY T.F.C. Introduction to Quantitative Genetics. 4.ed. Addison Wesley Longman, Harlow, 1996, 484p.

FAO. FAOSTAT - Base de dados estatísticos da Food and Agriculture Organization of the United Nations. Roma. Disponível em <www.faostat.fao.org>. Acesso em 14 de dezembro de 2007.

FERREIRA, G.B.; FERNANDES, H.D. Parâmetros genéticos para as características produtivas em bovinos da raça Holandesa no estado de Goiás. Revista Brasileira de Zootecnia, v.29, n.2, p.421-426, 2000. Disponível em: <http://www.scielo.br/ scielo.ph p ? script =sci_art text \& pid = S 1516 $35982000000200015 \& \operatorname{lng}=$ en $\& n r m=i s o \& t \operatorname{lng}=p t>$. Doi: 10.1590/S1516-35982000000200015.

FERREIRA, W.J. et al. Avaliação genética de bovinos da raça holandesa usando a produção de leite no dia do controle. Revista Brasileira de Zootecnia, v.32, n.2, p.295-303, 2003. Disponível em: <http:// www.scielo.br/scielo.php?script=sci_arttext\&pid=S151635982003000200007\&lng=en\&nrm=iso\&tlng=pt>. Doi: 10.1590/ S1516-35982003000200007.

FREITAS, A.F. et al. Parâmetros genéticos para produção de leite e gordura nas três primeiras lactações de vacas Holandesas. Revista Brasileira de Zootecnia, v.30, n.3, p.709-713, 2001. Disponível em: <http://www.scielo.br/scielo.php?script=sci_arttext\&pid=S151635982001000300015\&lng=en\&nrm=iso\&tlng=pt>. Doi: 10.1590/ S1516-35982001000300015.
FREITAS, A.F. Persistência da lactação: uma referência absoluta. Publicado em 18/03/2005 por Romualdo Venâncio - Revista Balde Branco. Disponível em: <http:// www.rehagro.com.br/siterehagro/publicacao.do?cdnoticia=1001. Acesso em: 13 jan. 2008.

GENGLER, N. Persistency of lactation yields: A review. Proc. Int. Workshop on Genetic Improvement of functional Traits in Cattle. Interbull Bull. v.12, p.97-102, 1996.

GENGLER, N. et al. Estimation of (co)variance functions of test day yields in first and later lactation of United States Holstein cows. Proc. Int. Workshop on Genetic Improvement of functional Traits in Cattle. Interbull Bull, v.22, p.69-73, 1999.

JAKOBSEN, J.H. et al. Genetic parameters for milk production and persistency for Danish Holstein estimated in random regression models using REML. Journal of Dairy Science, v.85, n.6, p.1607-1616, 2002.

JAMROZIK, J.; SCHAEFFER. L.R. Estimates of genetic parameters for a test day model with random regression for yield traits of first lactation Holstein. Journal of Dairy Science, v.80, n.4, p.762-770, 1997.

JAMROZIK, J. et al. Approximate accuracies of prediction from random regression models. Livestock Production Science, 66:85-92. 2000. Disponível em: <http://www.sciencedirect.com/ science?_ob=ArticleURL\&_udi=B6T9B-40Y3SYS$8 \&$ \&user $=687358 \&$ \&doc $=1 \&$ \& $\mathrm{fmt}=\&$ \&_orig $=$ search\&_so $\mathrm{rt}=\mathrm{d} \& \mathrm{v}$ i e $\mathrm{w}=\mathrm{c} \& \quad$ a c c t $=$ C $0000037899 \&$ \& v e r s i o n $=1 \&$ \& r l V e r s i o $n=0 \&$ userid=687358\&md5=92bd61500e21b7c2dc7f247571de4aa6 $>$. Doi: 10.1016/S0301-6226(00)00158-5.

KISTEMAKER, G.J. Comparison of persistency definitions in random regression test day models. Interbull Bull, v.30, p.96-98, 2003.

LIN, C.Y.; TOGASHI, K. Simultaneous improvement of lactation milk and persistence. In: WORLD CONGRESS OF GENETICS APPLIED LIVESTOCK PRODUCTION, 7., 2002, Montpellier, France. Proceedings... Montpellier: Communication. 09-39, 2002. (CD-ROM).

LUDWICK, T.M.; PETERSEN, W.E. A measure of persistency of lactation of dairy cattle. Journal of Dairy Science, v.26, p.439-445, 1943. Disponível em: <http://www.sciencedirect.com/ science?_ob=ArticleURL\&_udi=B6T9B-4FSFXJS$1 \&$ \&user $=687358 \&$ _rdoc $=1 \&$ \& fmt $=$ \&_orig $=$ search $\&$ _sort $=\mathrm{d}$ $\&$ vi e w $=$ c \&_a c c t $=$ C $000037899 \&$ _ versio $\mathrm{n}=1 \& \&_{-}$u r l V e r s i o $n=0 \&$ _ u s e r i d $=6$ 87358\&md5=aee8f6439af74c0696f1a1a9d9ca28a1>. Doi: 10.1016/j.livprodsci.2004.11.042.

MADSEN, O. A comparison of some suggested measures of persistency of milk yield in dairy cows. Animal Production, v.20, p.191-197, 1975.

MARION, A.E. et al. Estudo da heterogeneidade das variâncias para as características produtivas de rebanhos da raça Holandesa no Rio Grande do Sul. Revista Brasileira de Zootecnia, v.30, n.6, p.1995-2001, 2001. 
MEYER, K. “DXMRR” - A program to estimate covariance functions for longitudinal dada by Restricted Maximum Likelihood. In: WORLD CONGRESS OF GENETICS APPLIED LIVESTOCK PRODUCTION, 6., 1998, Armidale, Australia, Proceedings... Armidale: University of New England, v.27, p.465-466, 1998

MELO, C.M.R. et al. Parâmetros genéticos para as produções de leite no dia do controle e da primeira lactação de vacas da raça holandesa. Revista Brasileira de Zootecnia, v.34, n.3, p.796-806, 2005. Disponível em: <http://www.scielo.br/ scielo.php? script =sci_art text \& pid = S 1516 $35982005000300011 \& \operatorname{lng}=$ en $\& n r m=i s o \& t \operatorname{lng}=p t>$. Doi: 10.1590/S1516-35982005000300011.

MUIR, B.L. et al. Genetic relationships between persistency and reproductive performance in first lactation Canadian Holsteins. Journal of Dairy Science.v.87, n.9, p.3029-3037, 2004 .

REKAYA, R. et al. Hierarchical nonlinear model for persistency of milk yield in the first three lactation of Holsteins. Livestock Production Science, v.68, p.181-187, 2001. Disponível em: $<\mathrm{h} \mathrm{t} \mathrm{t} \mathrm{p} \mathrm{:} \mathrm{/} \mathrm{/} \mathrm{w} \mathrm{w} \mathrm{w.} \mathrm{s} \mathrm{c} \mathrm{i} \mathrm{e} \mathrm{n} \mathrm{c} \mathrm{e} \mathrm{d} \mathrm{i} \mathrm{r} \mathrm{e} \mathrm{c} \mathrm{t.} \mathrm{c} \mathrm{o} \mathrm{m}$ science?_ob=ArticleURL\&_udi=B6T9B-42H1RS2 $8 \&$ \&user $=687358 \&$ \&doc $=1 \&$ \&mt $=\&$ \&orig $=$ search \&_sort $=$ d \&vie w = c \&_a c c t $=$ C $000037899 \&$ _ ver si o n $=1 \&$ _ urlVersion=0\&_userid=687358\&md5=8f2591c39387115be266e76d6ca6ac6c $>$. Doi: 10.1016/S0301-6226(00)00239-6.

SANDERS, H.G. The analysis of the lactation curve into maximum yield and persistency. Journal of Agricultural Science Cambridge, v.20, p.145-185, 1930.
SHAHRBABAK, M.M. Feasibility of random regression models for Iranian Holstein test day records. 1997. 138p. Thesis (PhD) - University of Guelph, Guelph, Canada.

STRABEL, T.; JAMROZIK, J. The effect of incorrect estimated variance covariance components on genetic evaluation of dairy cattle with random regression models. In: WORLD CONGRESS OF GENETICS APPLIED LIVESTOCK PRODUCTION, 7., 2002, Montpellier, France. Proceedings... Montpellier: Communication. 01-09, 2002. (CD-ROM).

TEKERLI, M. et al. Factors affecting the shape of lactation curves of Holstein cows from the Balikesir province of Turkey. Journal of Dairy Science, v.83, n.6, p.1381-1386, 2000.

VAN DER LINDE, R. et al. Estimation of genetic parameters for persistency of milk production in dairy cattle. Proc. Int Workshop on Genetic Improvement of functional Traits in Cattle. Interbull Bull. v.25, p.113-116, 2000.

WEBER, T. et. al. Coeficientes de herdabilidade e correlações genéticas para as produções de leite e de gordura, em diferentes níveis de produção, para raça holandesa no estadado do Rio Grande do Sul. Revista Brasileira de Zootecnia, v.34 n.2 p.514-519, 2005. Disponível em: <http://www.scielo.br/ scielo.php? script =sci_art text \& pid = S 1516 35982005000200019\&lng=en\&nrm=iso\&tlng=pt>. Doi: 10.1590/S1516-35982005000200019.

WOOD, P.D.P. Algebraic model of the lactation curve in cattle. Nature, v.216, p.164-165, 1967. Disponível em: <http:// www.nature.com/doifinder/10.1038/216164a0>. Doi: 10.1038/ 216164a0 